\title{
Current status and therapeutic considerations of hypertension in the elderly
}

\author{
Ju-Hee Lee ${ }^{1,}$, Kwang-Il Kim ${ }^{2,}$, and Myeong-Chan Cho ${ }^{3}$
}

\author{
${ }^{1}$ Division of Cardiology, Department \\ of Internal Medicine, Chungbuk \\ National University Hospital, \\ Cheongju; ${ }^{2}$ Department of Internal \\ Medicine, Seoul National University \\ Bundang Hospital, Seongnam; \\ ${ }^{3}$ Department of Internal Medicine, \\ Chungbuk National University \\ College of Medicine, Cheongju, \\ Korea
}

As the prevalence of hypertension in the elderly population is increasing, information regarding the characteristics, optimal blood pressure targets, and special considerations for elderly hypertensive patients is needed to improve clinical outcomes. Various factors should be considered when managing hypertension in elderly patients, and there are many controversial issues and conflicting results related to the optimal treatment of hypertension in the elderly. In this review, we provide an overview of the epidemiology and characteristics of elderly hypertensive patients and discuss the optimal treatment of hypertension in elderly people.

Keywords: Aged; Hypertension; Epidemiology; Therapy

\section{Correspondence to Myeong-Chan Cho, M.D.}

Department of Internal Medicine, Chungbuk National University College of Medicine, 1 Chungdae-ro, Seowon-gu, Cheongju 28644, Korea Tel: +82-43-269-6356, Fax: +82-43-269-6354, E-mail: mccho@cbnu.ac.kr https://orcid.org/0000-0002-0047-0227

*These authors contributed equally to this work.

This paper was contributed by The Korean Society of Hypertension.
Received: June 14, 2019

Accepted: June 21, 2019 increased from 7.6 million in 1998 to 11.7 million in 2016 owing to the rapidly aging population (Fig. 1).

Korea has officially become an aged society, with an elderly population of over $14 \%$ in 2017 [2]. The youth population (aged o to 14) and the working-age population (aged 15 to 64) are decreasing yearly, whereas the elderly population (aged 65 or older) is increasing. Korea is estimated to become a 'super-aged' society with an elderly population of more than $20 \%$ in 2026 , and the elderly population will continue to grow until 2060, especially the portion comprising people aged 75 or older [2].

As the elderly population grows, the socioeconomic burden is also increasing. Medical costs associated with the elderly account for about half of Korea's total med- 


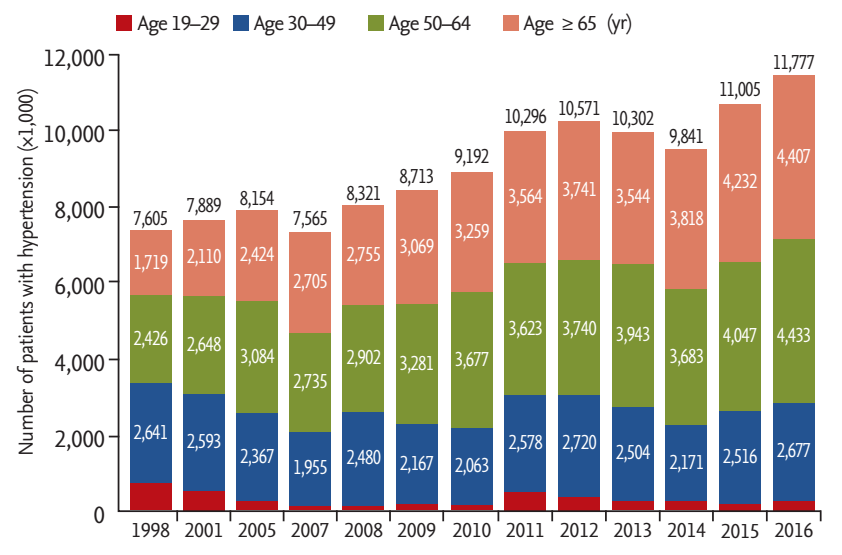

Figure 1. The prevalence of hypertension increases gradually with age. Although the prevalence of hypertension has remained relatively constant over the past 20 years, the number of hypertensive patients has steadily increased from 7.6 million in 1998 to 11.7 million in 2016 owing to the rapidly aging Korean population. Data from the 1998 to 2016 Korean National Health and Nutrition Examination Survey are shown. Adapted from Korean Society Hypertension (KSH) et al. [1], according to the Creative Commons License.

ical costs, and this proportion is expected to increase further [3]. Heart disease and cerebrovascular disease are the and and 3rd leading causes, respectively, of death in Korea, and hypertensive disease is the gth leading cause of death [4]. Death from circulatory issues, including hypertensive disease, ischemic heart disease, and cerebrovascular disease, has increased dramatically in the elderly population [4]. As the most important modifiable risk factor for cardiovascular and cerebrovascular diseases, the early diagnosis and proper control of hypertension are critically important in the elderly population.

Hypertension in the elderly differs from hypertension in young people in several important respects, and the management of elderly hypertension is the subject of controversy. In this review, we provide an overview of the epidemiology and characteristics of elderly hypertensive patients and discuss the optimal treatment for hypertension in elderly individuals.

\section{EPIDEMIOLOGY OF HYPERTENSION IN EL- DERLY KOREANS}

The Hypertension Epidemiology Research Group of the Korean Society of Hypertension (KSH) recently analyzed two national representative datasets, the 1998 to 2016 Korean National Health and Nutrition Examination Survey (KNHANES) dataset and the 2002 to 2016 National Health Insurance (NHI) Big Data, and published the Korea Hypertension Fact Sheet in 2018 [1]. Among hypertensive patients, the proportion of elderly patients (aged 65 years or older) has increased rapidly. In 2016, 37.4\% of hypertensive patients were aged 65 years or older (Fig. 1); the prevalence of hypertension also increased with age in both sexes, to more than $60 \%$ in males and more than $70 \%$ in females (Fig. 2A). The level of awareness and treatment for hypertension are continuously increasing, and are much higher in the elderly, particularly those aged 70 years or older (Fig. 2 B and 2 C). On the other hand, the control of hypertension does not differ greatly between the young and the elderly (Fig. 2D).

Our unpublished work analyzed the 2016 Korea National Health Insurance Claims Database and found that comorbidities were more common in hypertensive patients aged 65 years or older than aged less than 65 years. Among elderly hypertensive patients, $52.6 \%$ of patients had dyslipidemia, $29.0 \%$ had diabetes, $15.8 \%$ had ischemic heart disease, and $7.9 \%$ had had a stroke. The Charlson Comorbidity Index (CCI) [5] is used to score comorbidities based on ICD codes, and the CCI increased annually in all hypertensive patients from 2006 to 2016. The number of patients with multiple comorbidities and high CCI scores has also increased yearly, and has increased more rapidly in hypertensive patients aged 65 years or older (Fig. 3). Patients with multiple comorbidities $(\mathrm{CCI} \geq 3)$ are more prevalent in the elderly population, and more than $40 \%$ of elderly hypertensive patients had high CCI scores $\geq 3$. Regarding anti-hypertensive drug prescriptions, among elderly hypertensive patients, $48 \%$ of patients received monotherapy and $47 \%$ of patients received dual combination therapy (Fig. 4A). Calcium channel blockers (CCBs) were the most frequently prescribed anti-hypertensive treatment in elderly hypertensive patients (prescribed for about $47.7 \%$ of patients), followed by angiotensin receptor blockers (ARBs) (35.5\% of patients) (Fig. 4B). Among patients who received dual combination therapy, renin-angiotensin system blockers (RASBs) combined with CCBs were the most commonly prescribed (48.6\%), followed by RASBs and diuretics (27.3\%). 

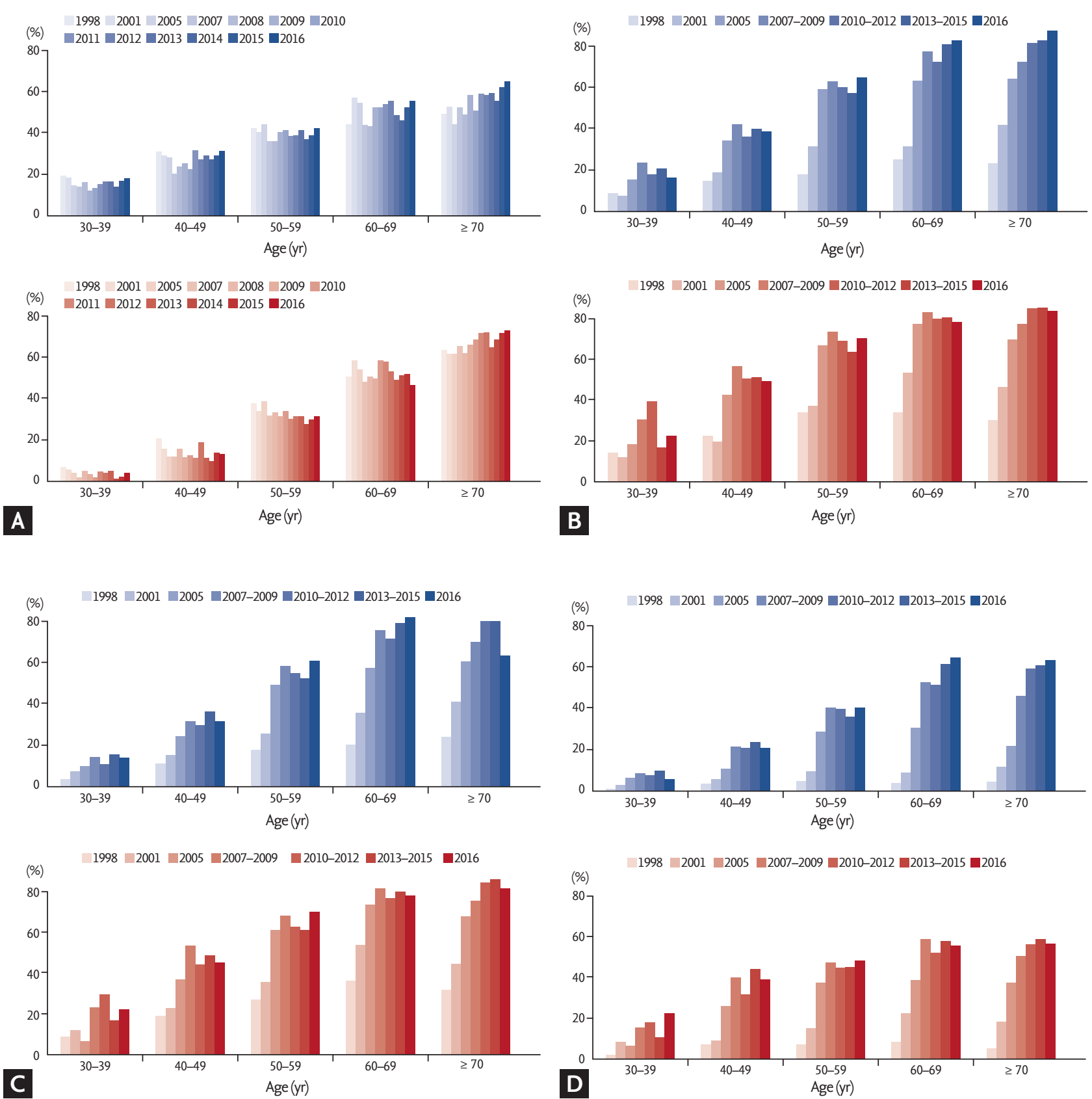

Figure 2. The awareness, treatment, and control rates of hypertension are generally improving. (A) According to data from Korean National Health and Nutrition Examination Survey (KNHANES), the awareness rate among hypertension patients increased from $25 \%$ in 1998 to $65 \%$ in 2016 . (B) The treatment rate also increased from $22 \%$ in 1998 to $61 \%$ in 2016 . (C) During the same period, the control rate increased significantly from $5 \%$ to $44 \%$ among all patients with hypertension, and (D) from $24 \%$ to $71 \%$ among treated hypertensive patients, according to KNHANES data. Blue column, male; red column, female. Adapted from Korean Society Hypertension (KSH) et al. [1], according to the Creative Commons License.

\section{CHARACTERISTICS OF HYPERTENSION IN THE ELDERLY}

Aging is associated with alterations in the structural and functional properties of large and small arteries, including arterial diameter, wall thickness, wall stiffness, and endothelial function [6-8]. As a consequence of increased arterial stiffness throughout the aging pro- 


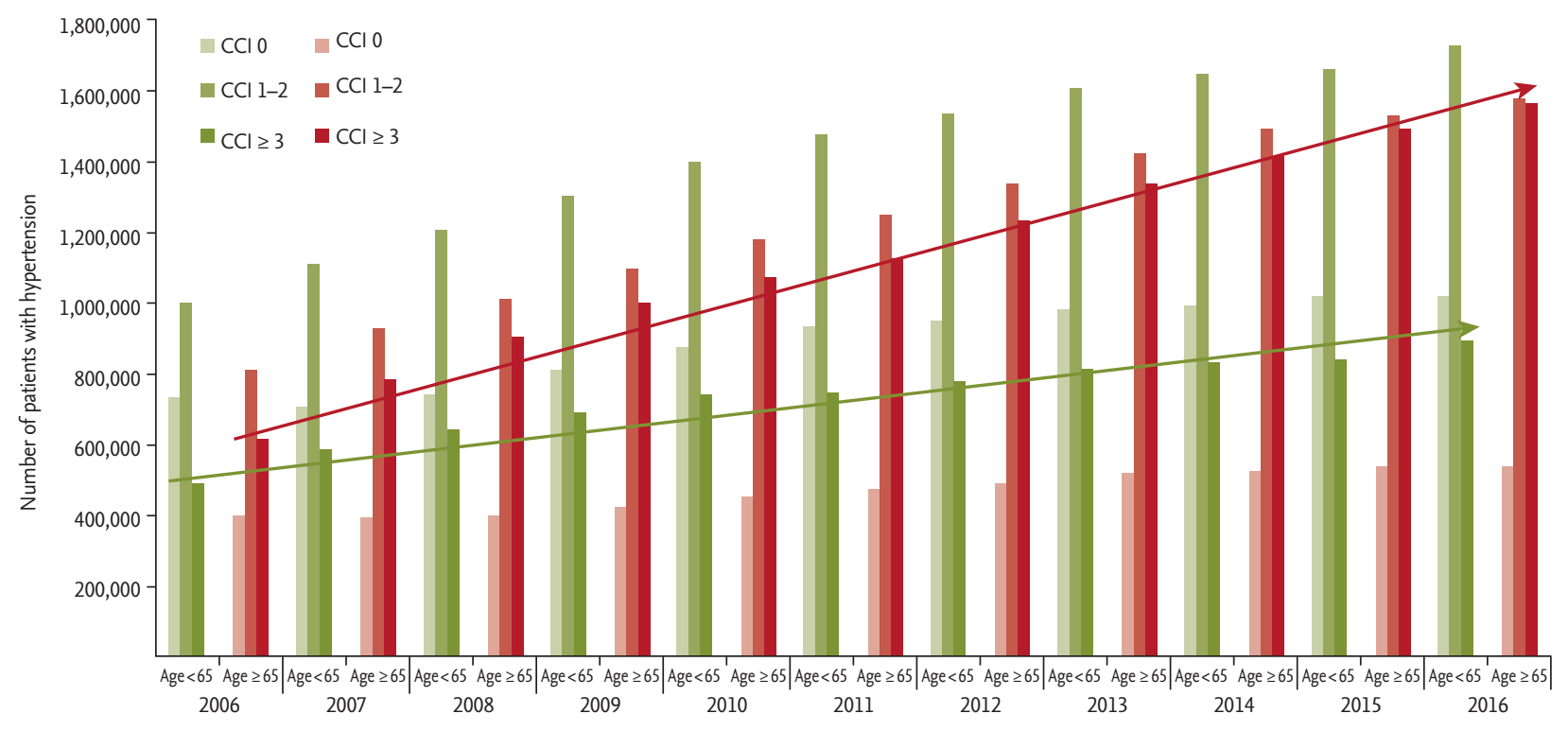

Figure 3. Comorbidities in elderly hypertensives. Patients with multiple comorbidities increased annually in all age groups, and increased more rapidly in elderly hypertensives. CCI, Charlson Comorbidity Index.
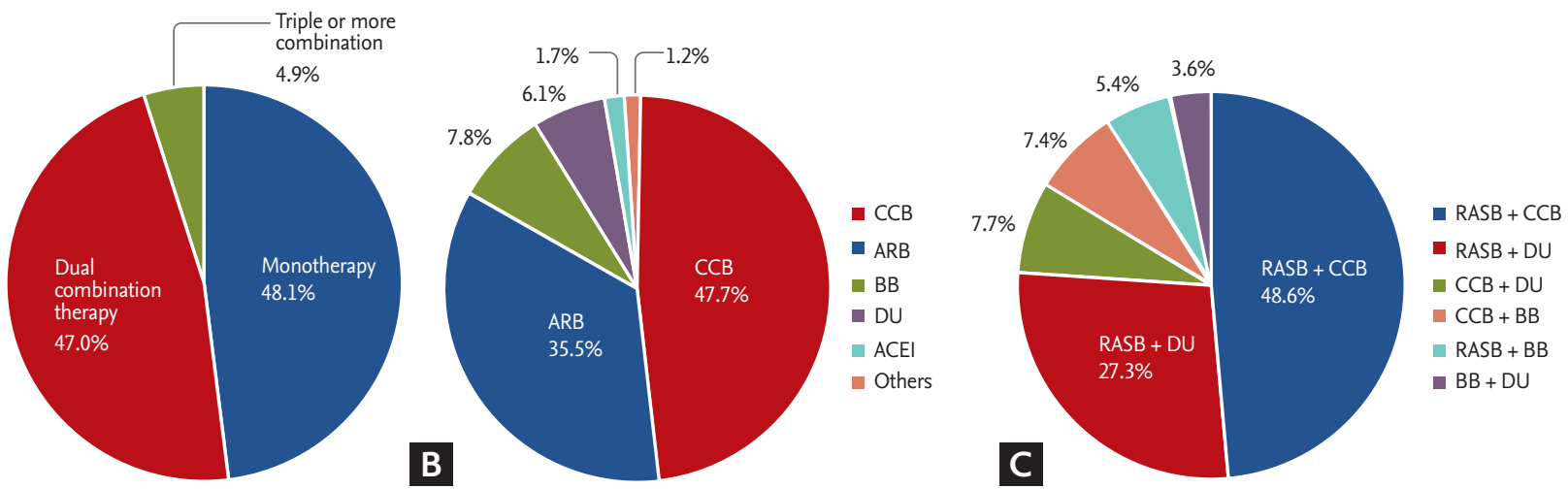

Figure 4. Antihypertensive drug prescriptions among hypertensive patients aged $\geq 65$ years. More than $95 \%$ of elderly hypertensive patients are treated by either monotherapy or dual combination therapy. (A) Currently, single pill combinations are most often prescribed for the elderly hypertensives to achieve better drug adherence. (B) Calcium channel blocker (CCB) and angiotensin receptor blocker (ARB) are the two most commonly used antihypertensive drugs for elderly hypertensives, followed by beta blocker (BB) and diuretic (DU). (C) Among patients who received dual combination therapy, combined renin-angiotensin system blocker (RASB) and CCB treatment is the most common prescription, followed by combined RASB and DU treatment. ACEI, angiotensin converting enzyme inhibitor.

cess, pulse wave velocity increases and reflected waves return faster [9]. Therefore, the amplitude of the sum of forward and reflected waves increases, as well as systolic blood pressure (BP) and pulse pressure. These are typical phenotypes of hypertension in the elderly. The exact pathophysiologic mechanism of such alterations remains unclear. However, structural alterations in the vascular wall, including an increased collagen content, decreased elastin density, decreased vascular smooth muscle cell nuclei, and increased fibroblasts, are likely contributors $[10,11]$. In addition, impaired endothelial function and vasodilation, nitric oxide deficiency, oxidative stress, small arterial remodeling, and maladaptation of the heart contribute to the development of hy- 
pertension in the elderly $[11,12]$.

There are a number of diagnostic challenges in elderly hypertensive patients. Pseudohypertension, a condition characterized by a significant discrepancy between intra-arterial BP and arm-cuff BP, is relatively common [13]. Pseudohypertensive patients usually exhibit minimal target organ damage despite a high arm-cuff BP, and they frequently experience postural hypotension despite anti-hypertensive therapy. In such cases, BP should be measured at alternative sites such as the wrist or finger [14]. Because of inter-measurement variability in $\mathrm{BP}$ readings, two or more readings should be taken initially in elderly hypertensive patients. Measurement of both sitting and standing BP is also recommended since orthostatic hypotension is encountered in up to $30 \%$ of elderly patients [14]. Because the 'white-coat' phenomenon is more prominent and nocturnal BP dipping is less pronounced in the elderly population, out-of-office BP measurement in addition to automated office BP measurement is recommended as needed. The advantages of out-of-office BP measurement techniques, such as home BP monitoring and ambulatory BP monitoring, include the ability to identify patients with whitecoat or masked hypertension, which is common among the elderly.

Because these specific characteristics are closely associated with poor cardiovascular outcomes [15], meticulous care should always be taken when treating elderly hypertensive patients.

\section{SPECIAL CONSIDERATIONS IN ELDERLY HYPERTENSIVE PATIENTS}

Several specific factors should be considered for the treatment of elderly hypertensive patients. Elderly people often suffer from issues such as frailty, multiple comorbidities, polypharmacy, cognitive impairment, depression, disability, falls, dizziness, and syncope. All of these interrelated factors can interfere with the control of BP. Thus, elderly hypertensive patients are a heterogeneous group, and chronological age is not necessarily synonymous with biological age.

\section{Multimorbidity}

As patients age, the rate of comorbidities increases [16].
As described in the previous section, elderly patients usually have multiple comorbidities such as dyslipidemia, diabetes, ischemic heart disease, stroke, heart failure, sleep apnea, chronic lung disease, and chronic kidney disease. A comprehensive diagnostic work-up for multiple comorbidities is required to evaluate the medical condition of elderly hypertensive patients because such comorbidities are associated with poor clinical outcomes. As a result, multimorbidity is the primary contributor to the increased medical costs among the elderly. Moreover, multimorbidity is common in hypertensive patients, and is associated with poor BP control [17]. Accordingly, multimorbidity must absolutely be considered in the treatment of hypertension in the elderly population. Polypharmacy associated with multiple comorbidities has also been pointed out as a major factor in the low drug adherence and low hypertension control rate among elderly hypertensive patients.

\section{Frailty}

Frailty is a common geriatric syndrome characterized by increased risks of disability, hospitalization, and mortality [18]; it is also closely associated with biological age [19]. The assessment of frailty is important in determining the correct hypertension treatment for elderly persons since there are significant differences in daily activity, independent self-care ability, cardiovascular risk, and the risk of adverse events during hypertension treatment according to frailty status.

Kang et al. [20] analyzed older adults ( $\geq 65$ years of age) from the 5 th KNHANES conducted from 2010 to 2012 and classified the subjects as robust, pre-frail, and frail using the frailty index. In this analysis, the prevalence of hypertension was higher in frail elderly patients (67.8\%) than in pre-frail $(60.8 \%)$ or robust elderly patients (49.2\%) $(p<0.001)$. Furthermore, the frail elderly were more likely to be treated than pre-frail or robust elderly hypertensive patients $(p<0.001)$. However, the BP control rate was significantly lower in frail elderly patients compared with pre-frail or robust patients $(p=0.005)$. The association between frailty index and BP followed a U-shaped trend, which means that the frailty index was higher in subjects with a high or low BP. Frailty status was also identified as a significant predictor of poor BP control. Among elderly patients aged 75 years or older enrolled in the SPRINT [21,22], the incidence of serious 
adverse events was higher in frail patients than in fit (robust) or less fit (pre-frail) patients, although there was no difference between treatment arms [23]. Orthostatic hypotension is another characteristic of frail elderly hypertensive patients. The impaired baroreceptor sensitivity and reduced cardiovascular sensitivity to catecholamines among the elderly increase BP sensitivity [14]. The variability and sensitivity of BP can be an obstacle to intensive BP control, and appropriate caution should be taken in the treatment of hypertension in elderly individuals.

Because of these considerations, clinical decision-making for hypertension in the elderly should not depend on chronological age alone, but also take into account the global risk assessment for individual patients, including comorbidities, frailty, functional status, and the risk of orthostatic hypotension.

\section{Dementia}

Hypertension is an established risk factor for vascular dementia. Recent findings also highlight the role of hypertension in the pathogenesis of Alzheimer's disease. Decreased cerebral blood flow due to atherosclerosis resulting from long-standing hypertension may be a major biological pathway linking hypertension to cognitive decline and dementia. However, it is unclear whether antihypertensive therapy can slow the progress of cognitive impairment among elderly hypertensive patients. Previous studies revealed marginal beneficial effects of BP-lowering therapies on cognition, but were limited by factors such as short follow-up owing to early trial termination and modest effects of treatment [24]. Recently, the SPRINT-MIND trial was initiated to investigate the effects of intensive BP control (systolic BP target $<120$ $\mathrm{mmHg}$ ) on the rate of probable dementia and mild cognitive impairment compared with the standard systolic BP treatment goal of $<140 \mathrm{mmHg}$ [25]. The trial failed to identify a difference in the incidence of probable dementia between the intensive and standard BP control treatments. This study was terminated early and included fewer cases of dementia than expected, and may thus have been underpowered for this endpoint.

Interestingly, increased BP variability has also been reported to be significantly associated with white matter hyperintensities and brain atrophy, which are predisposing conditions for dementia, depression, and falls in the elderly [26]. Several mechanisms have been suggested for the association between BP variability and cognitive impairment in elderly individuals, including increased cerebral blood flow fluctuations, neurohumoral activation, endothelial dysfunction, inflammation, and oxidative stress. However, additional studies are needed to identify the exact mechanism underlying the effects of hypertension on cognitive function and the optimal hypertensive treatment regime for dementia prevention.

\section{OPTIMAL HYPERTENSION TREATMENT IN THE ELDERLY}

The optimal target BP and treatment approach in elderly patients has not yet been established. Concerns have been raised regarding the benefit of intensive BP control and the risk of adverse events in elderly patients.

The Hypertension in the Very Elderly Trial (HYVET) [27] enrolled a relatively robust 3,845 subjects with an average age of 83.6 years and baseline systolic BP of 173 $\mathrm{mmHg}$. Subjects were randomly assigned to the active treatment group or placebo group. During 1.8 years of median follow-up duration, the mean BP was 15.0/6.1 $\mathrm{mmHg}$ lower in the active treatment group than in the placebo group. Active treatment was associated with a $30 \%$ reduction in fatal or non-fatal stroke as a primary endpoint, a $39 \%$ reduction in death from stroke, a $21 \%$ reduction in death from any cause, and a $64 \%$ reduction in heart failure. Adverse events occurred less frequently in the active treatment group ( 358 vs. $448, p=0.001$ ). The frailty index score did not alter the benefits of antihypertensive therapy for the HYVET population, although this population was comprised mainly of robust subjects [28].

In contrast, the Japanese Trial to Assess Optimal Systolic Blood Pressure in Elderly Hypertensive Patients study [29], which focused on elderly hypertensive patients (aged 65 to 85 years), found no significant difference in the primary endpoints of cardiovascular disease and renal failure between the strict treatment group (systolic BP target $<140 \mathrm{mmHg}$ ) and mild treatment group $(140 \mathrm{mmHg} \leq$ systolic $\mathrm{BP}$ target $<160 \mathrm{mmHg}$ ) over 2 years of median follow-up. Moreover, there were some concerns that strict BP control in the most elderly patients may have adverse effects since patients aged 75 years or older were more likely to have severe cerebro- 
vascular disease when treated strictly.

The recently published pre-specified subgroup analysis of the Systolic Blood Pressure Intervention Trial in Older Adults [22], which compared the effects of intensive BP treatment (systolic BP target $<120 \mathrm{mmHg}$ ) and standard BP treatment (systolic BP target $<140 \mathrm{mmHg}$ ) in elderly patients, instigated debate over the optimal target BP in elderly patients. A total of 2,636 patients aged $\geq 75$ years were included in this study, and the primary endpoint was a composite of nonfatal myocardial infarction, acute coronary syndrome not resulting in myocardial infarction, nonfatal stroke, nonfatal acute decompensated heart failure, and death from cardiovascular causes. At a median follow-up of 3.14 years, there were significantly lower rates of the primary outcome (102 events vs. 148 events; hazard ratio, 0.66) and all-cause mortality (73 deaths vs. 107 deaths; hazard ratio, 0.67 ) in the intensive treatment group than in the standard treatment group. The rates of serious adverse events, including hypotension, syncope, electrolyte abnormalities, and acute kidney injury, were higher in the intensive treatment group, but the difference was not significant. Although there were higher event rates with increasing frailty in both treatment groups, the absolute event rates within each frailty category were significantly lower in the intensive treatment group.

Based on the conflicting results of previous studies, the optimal target BP in elderly hypertensive patients differs among hypertension guidelines from different countries (Table 1). The 2017 American College of Cardiology/American Heart Association (ACC/AHA) Guideline for the Prevention, Detection, Evaluation, and Management of High Blood Pressure in Adults [30] recommended initiating antihypertensive medication when the systolic BP exceeds $130 \mathrm{mmHg}$ in patients aged 65 years or older. The ACC/AHA guidelines further suggest that the risk/benefit profile of treatment should be assessed if patients have a high comorbidity burden and/or limited life expectancy. On the other hand, the 2018 European Society of Cardiology/European Society of Hypertension Guidelines for the Management of Arterial Hypertension [31] recommended that in older patients treated for hypertension, office BP should be lowered to a systolic value of 130 to $139 \mathrm{mmHg}$ and a diastolic value of 70 to $79 \mathrm{mmHg}$ if tolerated, but that treated $\mathrm{BP}$ values of $<130 / 70 \mathrm{mmHg}$ should be avoided. The $2018 \mathrm{KSH}$ guidelines for the management of hypertension recommended initiating antihypertensive treatment when the systolic BP reached $140 \mathrm{mmHg}$ or higher, with a target BP of less than $140 \mathrm{mmHg}$ in elderly hypertensive patients.

Our unpublished work analyzed more than 40,000 hypertensive patients from the NHI Big Data and demonstrated a U-shaped relationship between the achieved systolic BP and the risk of composite outcomes, including cardiovascular death, admission of stroke, myocardial infarction, heart failure, and death. The How to Optimize Elderly Systolic Blood Pressure (HOWOLD-BP) trial (KCToo03787) is an ongoing study sponsored by the Korea National Institute of Health to assess cardiovascular outcomes in elderly hypertensive patients aged 65 or older according to a target systolic $\mathrm{BP}<130 \mathrm{mmHg}$ (intensive treatment group) and $<140 \mathrm{mmHg}$ (standard treatment group). The primary endpoint is a composite of acute coronary syndrome (including myocardial infarction and unstable angina), stroke, heart failure, and cardiovascular death. In addition to data on cardiovascular events and adverse events, the study will also collect and analyze data for the assessment of frailty, cognitive function, and quality of life. A total of 3,176 subjects will be enrolled in this study and will be followed for more than 6 years. The results of the HOWOLD-BP study are

Table 1. Comparison of treatment thresholds for hypertension and target BP in elderly hypertensive patients (age $\geq 65$ years) among various guidelines.

\begin{tabular}{llll}
\hline Variable & 2017 ACC/AHA guideline & 2018 ESC/ESH guideline & 2018 KSH guideline \\
\hline Treatment threshold, $\mathrm{mmHg}$ & $\geq 130$ (systolic BP) & $\geq 140 / 90$ in 65-79 years & $\geq 140 / 90$ \\
& & $\geq 160 / 90$ in $\geq 80$ years & $<140$ (systolic BP) \\
\hline
\end{tabular}

BP, blood pressure; ACC/AHA, American College of Cardiology/American Heart Association; ESC/ESH, European Society of Cardiology/European Society of Hypertension; KSH, Korean Society Hypertension. 
expected to provide robust evidence for the optimal target BP in elderly hypertensive patients and risk/benefit profiles for treatment regimes.

\section{CONCLUSIONS}

The prevalence of hypertension in the elderly is continuously increasing in Korea, in which about $40 \%$ of total hypertensive patients are aged 65 years or older. Although the awareness and treatment rates of hypertension are increasing, the condition remains poorly controlled in the elderly.

Various factors should be considered in the treatment of elderly hypertensive patients. For example, elderly hypertension may be related to vascular aging and cardiovascular remodeling. Many elderly hypertensive patients have multiple comorbidities, which should also be considered when determining the target $\mathrm{BP}$ and selecting antihypertensive drugs. Because tolerance for antihypertensive treatments and the risk of adverse events (e.g., orthostatic hypotension, syncope, falls, kidney injury, and electrolyte imbalances) can be affected by a patient's frailty and cognitive function, these factors should be assessed when initiating treatment.

There are many conflicting results on the optimal target BP in elderly hypertensive patients, and discrepancies exist among the guidelines for hypertension management. More evidence is needed to establish the optimal target BP in elderly patients. The ongoing HOWOLD-BP trial is expected to answer remaining questions, including those related to the optimal target $\mathrm{BP}$, frailty, BP control, quality of life, and cognitive function in elderly hypertensive patients.

\section{Conflict of interest}

No potential conflict of interest relevant to this article was reported.

\section{Acknowledgments}

This research was supported by a fund (2018-ER6303-00) by Research of Korea Centers for Disease Control and Prevention.

\section{REFERENCES}

1. Korean Society Hypertension (KSH); Hypertension Epidemiology Research Working Group, Kim HC, Cho MC. Korea hypertension fact sheet 2018. Clin Hypertens 2018;24:13.

2. Korean Statistical Information Service. 2017 Population and housing census [Internet]. Daejeon (KR): Statistics Korea, 2018 [cited 2019 Jun 21]. Available from: http://kosis.kr.

3. Korean Statistical Information Service. Medical service usage statistics by region [Internet]. Daejeon (KR): Statistics Korea, 2018 [cited 2019 Jun 21]. Available from: http:// kosis.kr.

4. Korean Statistical Information Service. 2017 Cause of death statistics [Internet]. Daejeon (KR): Statistics Korea, 2018 [cited 2019 Jun 21]. Available from: http://kosis.kr.

5. Charlson ME, Pompei P, Ales KL, MacKenzie CR. A new method of classifying prognostic comorbidity in longitudinal studies: development and validation. J Chronic Dis 1987;40:373-383.

6. Najjar SS, Scuteri A, Lakatta EG. Arterial aging: is it an immutable cardiovascular risk factor? Hypertension 2005;46:454-462.

7. Oh YS. Arterial stiffness and hypertension. Clin Hypertens. 2018;24:17.

8. Kim KI, Cho YS, Choi DJ, Kim CH. Optimal treatment of hypertension in the elderly: a Korean perspective. Geriatr Gerontol Int 2008;8:5-11.

9. O’Rourke MF, Adji A. An updated clinical primer on large artery mechanics: implications of pulse waveform analysis and arterial tonometry. Curr Opin Cardiol 2005;20:275281.

10. Lakatta EG. Arterial and cardiac aging: major shareholders in cardiovascular disease enterprises: Part III: cellular and molecular clues to heart and arterial aging. Circulation 2003;107:490-497.

11. Xu X, Wang B, Ren C, et al. Age-related impairment of vascular structure and functions. Aging Dis 2017;8:590610.

12. Nilsson PM. Early vascular aging (EVA): consequences and prevention. Vasc Health Risk Manag 2008;4:547-552.

13. Zweifler AJ, Shahab ST. Pseudohypertension: a new assessment. J Hypertens 1993;11:1-6.

14. Kjeldsen SE, Stenehjem A, Os I, et al. Treatment of high blood pressure in elderly and octogenarians: European Society of Hypertension statement on blood pressure tar- 
gets. Blood Press 2016;25:333-336.

15. Kario K. New insight of morning blood pressure surge into the triggers of cardiovascular disease-synergistic resonance of blood pressure variability. Am J Hypertens 2016;29:14-16.

16. Barnett K, Mercer SW, Norbury M, Watt G, Wyke S, Guthrie B. Epidemiology of multimorbidity and implications for health care, research, and medical education: a cross-sectional study. Lancet 2012;380:37-43.

17. Wong MC, Wang HH, Cheung CS, et al. Factors associated with multimorbidity and its link with poor blood pressure control among 223,286 hypertensive patients. Int J Cardiol 2014;177:202-208.

18. Klein BE, Klein R, Knudtson MD, Lee KE. Frailty, morbidity and survival. Arch Gerontol Geriatr 2005;41:141-149.

19. Mitnitski A, Collerton J, Martin-Ruiz C, et al. Age-related frailty and its association with biological markers of ageing. BMC Med 2015;13:161.

2o. Kang MG, Kim SW, Yoon SJ, Choi JY, Kim KI, Kim CH. Association between frailty and hypertension prevalence, treatment, and control in the elderly Korean population. Sci Rep 2017;7:7542.

21. SPRINT Research Group, Wright JT Jr, Williamson JD, et al. A randomized trial of intensive versus standard blood-pressure control. N Engl J Med 2015;373:2103-2116.

22. Williamson JD, Supiano MA, Applegate WB, et al. Intensive vs standard blood pressure control and cardiovascular disease outcomes in adults aged $\geq 75$ years: a randomized clinical trial. JAMA 2016;315:2673-2682.

23. Supiano MA, Williamson JD. Applying the systolic blood pressure intervention trial results to older adults. J Am Geriatr Soc 2017;65:16-21.

24. Peters R, Beckett N, Forette F, et al. Incident dementia and blood pressure lowering in the Hypertension in the Very Elderly Trial cognitive function assessment (HYVET-
COG): a double-blind, placebo controlled trial. Lancet Neurol 2008;7:683-689.

25. SPRINT MIND Investigators for the SPRINT Research Group, Williamson JD, Pajewski NM, et al. Effect of intensive vs standard blood pressure control on probable dementia: a randomized clinical trial. JAMA 2019;321:553561.

26. Jung HW, Kim KI. Blood pressure variability and cognitive function in the elderly. Pulse (Basel) 2013;1:29-34.

27. Beckett NS, Peters R, Fletcher AE, et al. Treatment of hypertension in patients 80 years of age or older. $\mathrm{N}$ Engl $\mathrm{J}$ Med 2008;358:1887-1898.

28. Warwick J, Falaschetti E, Rockwood K, et al. No evidence that frailty modifies the positive impact of antihypertensive treatment in very elderly people: an investigation of the impact of frailty upon treatment effect in the HYpertension in the Very Elderly Trial (HYVET) study, a double-blind, placebo-controlled study of antihypertensives in people with hypertension aged 80 and over. BMC Med 2015;13:78.

29. JATOS Study Group. Principal results of the Japanese trial to assess optimal systolic blood pressure in elderly hypertensive patients (JATOS). Hypertens Res 2008;31:21152127.

30. Whelton PK, Carey RM, Aronow WS, et al. 2017 ACC/AHA/ AAPA/ABC/ACPM/AGS/APhA/ASH/ASPC/NMA/PCNA guideline for the prevention, detection, evaluation, and management of high blood pressure in adults: a report of the American College of Cardiology/American Heart Association Task Force on clinical practice guidelines. Circulation 2018;138:e484-e594.

31. Williams B, Mancia G, Spiering W, et al. 2018 ESC/ESH guidelines for the management of arterial hypertension. Eur Heart J 2018;39:3021-3104. 